\title{
PARÂMETROS GENÉTICOS E ASSOCIAÇÃO DE METODOLOGIAS DE EUN NO MILHO SOB DIFERENTES DOSES DE NITROGÊNIO
}

\section{GENETICS PARAMETERS AND ASSOCIATION OF NUE METHODS IN MAIZE UNDER DIFFERENT NITROGEN LEVELS}

Edmar Vinícius de CARVALHO ${ }^{[1, *]}$ Flávio Sérgio AFFÉRR[ ${ }^{[2]}$ Joenes Mucci PELUZIO ${ }^{[1]}$, Eliane Aparecida ROTILI $^{[1]}$, Michel Antônio DOTTO ${ }^{[1]}$ e Lucas Alves de FARIA ${ }^{[1]}$

[1] Universidade Federal do Tocantins, campus Universitário de Gurupi. Rua Badejós, S/N Zona Rural. CEP $77402-970$ - Gurupi, TO - Brasil. email joenesp@uft.edu.br, elianerotili@yahoo.com.br, mdotto@gdmseeds.com elucasfaria_16@hotmail.com

[2] Universidade Federal de São Carlos, Centro de Ciências da Natureza, campus Lagoa do Sino. CEP 18295-990 - Buri, SP - Brasil. email flavioafferri@gmail.com

*Autor para correspondência: carvalho.ev@uft.edu.br

INFORMAÇÕES

Recebido em: 02/09/2015

Aceito em: 25/02/2016

Publicado em: 28/03/2016

Document Object Identifier

$10.18067 / j b f s . v 3 i 1.61$

Editor: V. H. G. Sales

jbfs@ifap.edu.br

ID JBFS2015061

Avaliação a cega por pares

Prot. 0612015R01

Prot. 0612015R02

Copyright: (C) 2016

JBFS all rights (BY NC SA)

\section{RESUMO}

O objetivo do trabalho foi estudar a associação de quatro metodologias de eficiência do uso do nitrogênio (EUN) e os parâmetros genéticos da produtividade grãos em dois grupos de genótipos de milho sob diferentes níveis de nitrogênio em cobertura, na safra 2012/13. Foram conduzidos 16 experimentos de campo no município de Gurupi-TO. Cada grupo de genótipo foi avaliado em datas de semeadura distintas e com diferentes níveis de fornecimento de $\mathrm{N}$ em cobertura. $\mathrm{O}$ delineamento experimental foi o de blocos ao acaso com três repetições. A produtividade de grãos foi avaliada após a maturação fisiológica (estágio R6) e posteriormente, estimados quatro índices de eficiência/estresse ao nitrogênio. Os coeficientes de correlação de Pearson, estimado entre os índices, foram todos significativos $(p<0,01)$. A herdabilidade da produtividade de grãos foi de $54,4 \%$, na média das datas de semeadura, e de $60,4 \%, 50,9 \%, 51,2 \%$ considerando os níveis baixos, médio e alto de fornecimento de $\mathrm{N}$ em cobertura, respectivamente. Existe a possibilidade do uso de ambientes com baixo fornecimento de $\mathrm{N}$ na seleção de genótipos superiores $\mathrm{e}$ eficientes quando a produtividade de grãos e, ainda, baseado nos resultados o índice "Low $\mathrm{N}$ Index" é mais adequado.

Palavras-chave: correlação, melhoramento, herdabilidade, Tocantins, Zea mays L.

ABSTRACT - This work aimed to study the association of four nitrogen use efficiency (NUE) methods and the genetic parameters of grain weight in two groups of maize genotypes, under different levels of nitrogen supply, in the season 2012/13. 16 field experiments were carried out in the city of Gurupi, Tocantins, Brazil. Each genotype group was evaluated in different seeding date, and each one was tested with different levels of nitrogen supply. In all experiments the experimental design was completely randomized blocks with three repetitions. The following trait was evaluated after stage R6: grain yield (GY), and after, four indices of efficiency/stress to nitrogen were estimated. The Pearson correlation coefficients, estimated among the indices, were all significant $(p<0.01)$. Among the seeding dates, the average heritability of GY was $54.4 \%$ and among the levels of nitrogen supply, the following values were observed: $60.4 \%$ (low N); $50.9 \%$ (medium N); $51.2 \%$ (high N). There is the possibility of the use of environments with lower nitrogen supply in the search for superior and more efficient genotypes for the GY, and based on our results, the Low $\mathrm{N}$ index is more adequate.

Keywords: breeding, correlation, heritability, Tocantins, Zea mays L

Financiamento: CNPq e CAPES (Bolsa de Doutorado ao primeiro autor).

Conflito de interesse: Os autores declaram que não há conflito de interesse.

Como referir esse documento (ABNT):

CARVALHO, E.V.; AFFÉRRI, F.S.; PELUZIO, J.M.; ROTILI, E.A.; DOTTO, M.A.; FARIA, L.A. Parâmetros genéticos e associação de metodologias de EUN no milho sob diferentes doses de nitrogênio. Journal of Bioenergy and Food Science, Macapá, v.3, n.1, p.3641, jan./mar., 2016. DOI:10.18067/jbfs.v3i1.61 


\section{INTRODUÇÃO}

O milho é uma das espécies cultivadas mais estudadas no mundo e isto se justifica pela sua importância na alimentação ${ }^{1}$ e como matéria-prima em diversas indústrias. $O$ sucesso desses estudos depende da variabilidade genética do atributo de interesse $^{2}$ a qual pode ser mensurada com o uso de um ou vários ambientes.

Com o aumento da população mundial, o aumento da produtividade em ambientes com estresse, por exemplo o nitrogenado, será necessário. ${ }^{3} \mathrm{O}$ nitrogênio, de acordo com Cirilo et al., ${ }^{4}$ é um dos fatores que limitam a produção vegetal em virtude da sua essencialidade para as plantas $^{2}$ e pelos impactos gerados pelo manejo inadequado dos fertilizantes. ${ }^{5}$

Na maioria dos solos, a disponibilidade de $\mathrm{N}$ é limitada (baixa), no entanto, é possível encontrar genótipos de milho produtivos nestas condições ${ }^{6}$. Por exemplo, Chen et al..$^{5}$ reportam que genótipos desenvolvidos em ambientes com baixa disponibilidade de $\mathrm{N}$ podem resultar em ganhos de $10 \%$ a $14 \%$ na produtividade de grãos.

Com relação a mensuração a eficiência do uso do $\mathrm{N}$, Wu et al. ${ }^{2}$ relatam a importância da

correta mensuração e que a busca por metodologias mais práticas é fundamental. Ainda, Combs e Bernardo ${ }^{7}$ relatam sobre a busca de metodologias mais rápidas e eficientes em relação as tradicionais.

O objetivo do trabalho foi estudar a associação de quatro metodologias de eficiência do uso do nitrogênio e os parâmetros genéticos da produtividade grãos em dois grupos de genótipos de milho sob diferentes níveis de nitrogênio em cobertura, na safra 2012/13.

\section{MATERIAL E MÉTODOS}

Na safra 2012/13, foram conduzidos 16 experimentos de campo em cinco áreas ( $A 1 ; A 2$; A3; A4; A5) localizadas no município de Gurupi-TO (11ㄴ3' S; 49-04' W; 280 m), em Latossolo Vermelho Amarelo, com clima do tipo B1wA'a' úmido com moderada deficiência hídrica, segundo a classificação de Köppen. Na Tabela 1, estão representados os resultados da análise de solo, na camada de $0-20 \mathrm{~cm}$, de cada uma das áreas em que foram conduzidos os experimentos e, também, a descrição resumida dos experimentos.

Tabela 1. Análise química e de textura do solo, na camada $0-20 \mathrm{~cm}$, e descrição resumida dos experimentos, em cada área, no ano safra 2012/13, Gurupi-TO, Brasil.

Table 1. Chemical analysis and soil texture in the $0-20 \mathrm{~cm}$ layer, and brief description of the experiments, in each area in the crop year 2012/13, Gurupi-TO, Brazil.

\begin{tabular}{|c|c|c|c|c|c|c|}
\hline \multirow{2}{*}{ Atributos } & \multicolumn{6}{|c|}{ Áreas } \\
\hline & $\mathrm{A} 1$ & $\mathrm{~A} 2$ & A3 & A4 & \multicolumn{2}{|c|}{ A5 } \\
\hline $\mathrm{pH}\left(\mathrm{CaCl}_{2}\right)$ & 5,40 & 4,70 & 5,10 & 5,30 & \multicolumn{2}{|c|}{5,20} \\
\hline $\mathrm{P}\left(\mathrm{mg} \cdot \mathrm{kg}^{-1}\right)$ & 6,40 & 1,80 & 3,70 & 4,70 & \multicolumn{2}{|c|}{10,40} \\
\hline $\mathrm{Ca}+\mathrm{Mg}\left(\mathrm{cmol} . \mathrm{dm}^{-3}\right)$ & 3,30 & 1,10 & 2,20 & 3,00 & \multicolumn{2}{|c|}{2,30} \\
\hline $\mathrm{H}+\mathrm{Al}\left(\mathrm{cmol} \cdot \mathrm{dm}^{-3}\right)$ & 1,70 & 2,40 & 2,30 & 2,70 & \multicolumn{2}{|c|}{2,30} \\
\hline $\mathrm{K}\left(\mathrm{cmol} \cdot \mathrm{dm}^{-3}\right)$ & 0,11 & 0,04 & 0,12 & 0,22 & \multicolumn{2}{|c|}{0,13} \\
\hline CTC $\left(\mathrm{cmol} . \mathrm{dm}^{-3}\right)$ & 5,12 & 3,55 & 4,63 & 5,94 & \multicolumn{2}{|c|}{4,76} \\
\hline $\mathrm{V}(\%)$ & 66,86 & 32,36 & 50,39 & 54,50 & \multicolumn{2}{|c|}{51,60} \\
\hline Matéria Orgânica (g.dm³) & 23,00 & 16,00 & 15,00 & 16,00 & \multicolumn{2}{|c|}{20,00} \\
\hline Argila $\left(\mathrm{g} . \mathrm{kg}^{-1}\right)$ & 340 & 300 & 300 & 300 & \multicolumn{2}{|c|}{340} \\
\hline Silte $\left(\mathrm{g} . \mathrm{kg}^{-1}\right)$ & 90 & 80 & 80 & 80 & \multicolumn{2}{|c|}{90} \\
\hline Areia $\left(\mathrm{g} . \mathrm{kg}^{-1}\right)$ & 570 & 620 & 620 & 620 & \multicolumn{2}{|c|}{570} \\
\hline Precipitação (mm) & 828 & 502 & 502 & 327 & 811 & 327 \\
\hline Data de semeadura & $11 / 12 / 2012$ & $28 / 01 / 2013$ & $06 / 02 / 2013$ & $13 / 03 / 2013$ & $30 / 10 / 2012$ & $06 / 03 / 2013$ \\
\hline Sistema de cultivo & $\mathrm{C}^{1}$ & $\mathrm{PD}^{2} / \mathrm{Co}^{3}$ & $\mathrm{PD}^{4}$ & $P D^{2} / I^{5}$ & $C^{1}$ & $1^{5}$ \\
\hline Quantidade de genótipos & 12 & 12 & 12 & 12 & 30 & 30 \\
\hline Quantidade de experimentos ${ }^{6}$ & 3 & 3 & 3 & 3 & 2 & 2 \\
\hline
\end{tabular}

${ }^{1}$ Sistema de Cultivo Convencional; ${ }^{2}$ Plantio Direto sob palhada de Feijão-Guandu (Cajanus cajan). ${ }^{3}$ Consórcio com $C$. cajan. ${ }^{4}$ Plantio Direto sob palhada de Braquiária (Brachiaria spp.). ${ }^{5}$ Irrigado. ${ }^{6}$ Representados pela dose de nitrogênio em cobertura.

${ }^{1}$ Conventional farming system; ${ }^{2}$ Tillage about straw Bean-Guandu (Cajanus cajan); ${ }^{3}$ Consortium with C. cajan; ${ }^{4}$ Tillage about straw of Brachiaria (Brachiaria spp.); ${ }^{5}$ Irrigated; ${ }^{6}$ Represented by the nitrogen dose in coverage.. 
Os experimentos de avaliação do grupo com 12 genótipos foram semeados em quatro áreas na seguinte ordem cronológica: A1 (11/12); A2 (28/01); A3 (06/02); A4 (13/03). Em cada semeadura foram implantados três experimentos, representados por três doses de nitrogênio em cobertura: 20, 80 e $140 \mathrm{~kg} \mathrm{ha}^{-1}$; aplicadas em dose única no estágio $\mathrm{V} 6$, sendo denominados de baixo, médio e alto $\mathrm{N}$ (quanto ao fornecimento), respectivamente. $\mathrm{O}$ grupo de genótipos foi composto por seis populações de polinização aberta, desenvolvidas por "top crosses" de linhagens com testador de base genética ampla e; seis genótipos comerciais. 0 delineamento experimental utilizado em cada experimento foi de blocos ao acaso com três repetições, sendo a parcela experimental constituída por duas linhas de cinco metros de comprimento, com espaçamento entre linhas de $0,75 \mathrm{~m}$. O número de plantas, após desbaste, foi de 50.000 plantas por hectare, exceto nos experimentos da área A4 (semeados em 13/03), que foi de 40.000 plantas por hectare.

$\mathrm{Na}$ área $\mathrm{A5}$, foram implantados quatro experimentos, sendo dois no período de safra verão $(30 / 10)$ e dois em período compreendendo semeadura tardia (06/03). Em cada período, foram utilizadas duas doses de nitrogênio em cobertura: 0 e $150 \mathrm{~kg} \mathrm{ha}^{-1}$; aplicada em dose única quando as plantas estavam entre no estágio V6, para diferenciar os experimentos, sendo denominados de baixo e alto $\mathrm{N}$ (quanto ao fornecimento), respectivamente. $\mathrm{O}$ grupo de genótipos foi composto por 24 populações de polinização aberta, desenvolvidas por "top crosses" de linhagens com testador de base genética ampla e; seis linhagens S5. O delineamento experimental foi de blocos ao acaso com três repetições, sendo a parcela experimental representada por uma linha de dois metros, com espaçamento entre linhas de 0,75 $\mathrm{m}$. O número de plantas, após o desbaste, foi de 50.000 plantas por hectare.

A adubação de semeadura foi realizada com $500 \mathrm{~kg} \mathrm{ha}^{-1}(5-25-15+0,5 \%$ de $\mathrm{Zn})$, no sulco de plantio. Foi realizada irrigação, por meio de aspersão convencional, nos experimentos semeados nas áreas A4 (13/03) e A5 (06/03), com turno de rega de três dias. Os demais tratos culturais foram efetuados assim que se fizeram necessários, seguindo as recomendações técnicas da cultura do milho. ${ }^{8}$
No estágio R6 (maturação fisiológica), foram colhidas as espigas das plantas representativas da parcela para avaliação da produtividade de grãos, em g planta ${ }^{-1}$, corrigida a $13 \%$ de umidade. Os dados obtidos foram utilizados para estimar os seguintes índices de eficiência/estresse ao nitrogênio: índice de estresse ao nitrogênio; eficiência agronômica do nitrogênio;"10 "Low N index"; ${ }^{11}$ e índice de sensibilidade ao estresse. ${ }^{12}$

Nos experimentos instalados nas áreas $A 1$ (11/12), A2 (28/01), A3 (06/02) e A4 (13/03) foram utilizados os seguintes intervalos de adubação nitrogenada em cobertura, na estimação dos índices: $140-20$ e $80-20 \mathrm{~kg} \mathrm{ha}^{-1}$. Nos demais, o intervalo usado foi de $150-0 \mathrm{~kg} \mathrm{ha}^{-1}$.

Nas análises estatísticas os experimentos foram agrupados de acordo com a data em que foram semeados. As análises estatísticas realizadas foram: (i) correlação linear entre os índices de eficiência/estresse ao nitrogênio em cada intervalo de $N$, com $n=90$ (30 genótipos $\times 3$ repetições) ou $\mathrm{n}=36 \quad(12$ genótipos $\mathrm{x} \quad 3$ repetições); (ii) herdabilidade em cada nível de $\mathrm{N}$ e; (iii) correlação fenotípica e genotípica entre os níveis de $\mathrm{N}$, com $\mathrm{n}=90$ (30 genótipos $\times 3$ repetições) ou $n=36$ (12 genótipos $\times 3$ repetições). A significância dos coeficientes foi verificada pelo teste $t(p<0,05)$.

\section{RESULTADOS E DISCUSSÃO}

Os coeficientes de correlação de Pearson, estimados entre os índices de eficiência/estresse ao nitrogênio foram significativos $(p<0,01)$ e maiores que 0,89 (valor absoluto) em todas análises realizadas (Tabela 2 ). Todos os coeficientes foram significativos pelo teste $t(p<0,01)$.

Valores negativos foram encontrados entre o índice "Low $\mathrm{N}$ index", proposto por Fishcer et al. ${ }^{10} \mathrm{e}$ os demais índices, no entanto, nos demais pares, o sinal foi positivo. Os quatro índices de eficiência/estresse ao nitrogênio estimados utilizam os valores de produtividade de grãos e fornecimento de $\mathrm{N}$ de diferentes maneiras nas suas fórmulas, porém os resultados obtidos apresentam concordância na avaliação dos genótipos. $O$ índice proposto por Fischer et al. ${ }^{11}$ possui como vantagem a não utilização da subtração na fórmula, e assim, sem possibilidade da obtenção de valores negativos o que facilita as análises estatísticas. 
Tabela 2. Coeficientes de correlação de Pearson entre os índices de eficiência/estresse ao nitrogênio estimados na avaliação de 12 genótipos de milho em quatro datas de semeadura (11/12; 28/01; 06/02; 13/03) e de 30 genótipos de milho em duas datas de semeadura (30/10; 06/03), Gurupi-TO, Brasil ano safra 2012/13.

Table 2. Pearson correlation coefficients between the levels of efficiency / stress to nitrogen estimated in the evaluation of 12 corn genotypes in four sowing dates (11/12; 28/01; 06/02; 13/03) and 30 corn genotypes in two sowing dates (30/10; 06/03), Gurupi-TO, Brazil, 2012/13 crop year.

\begin{tabular}{lllllll}
\hline \multirow{2}{*}{ Data Semeadura } & \multicolumn{7}{c}{ Alto N - Baixo N } \\
\cline { 2 - 7 } & IE2 $\times$ EA & IE2 x IE1 & IE2 x ISE & EA x IE1 & EA x IS & IE1 x IS \\
\hline $30 / 10$ & 1,00 & $-0,91$ & 0,91 & $-0,91$ & 0,91 & $-1,00$ \\
$06 / 03$ & 1,00 & $-0,89$ & 0,89 & $-0,89$ & 0,89 & $-1,00$ \\
$11 / 12$ & 1,00 & $-0,98$ & 0,98 & $-0,98$ & 0,98 & $-1,00$ \\
$28 / 01$ & 1,00 & $-0,96$ & 0,96 & $-0,96$ & 0,96 & $-1,00$ \\
$06 / 02$ & 1,00 & $-0,98$ & 0,98 & $-0,98$ & 0,98 & $-1,00$ \\
$13 / 03$ & 1,00 & $-0,98$ & 0,98 & $-0,98$ & 0,98 & $-1,00$ \\
\hline Data Semeadura & & \multicolumn{7}{c}{ Médio N-Baixo N } & $-0,97$ & 0,97 & $-1,00$ \\
$11 / 12$ & 1,00 & $-0,97$ & 0,97 & $-0,94$ & 0,94 & $-1,00$ \\
$28 / 01$ & 1,00 & $-0,94$ & 0,94 & $-0,98$ & 0,98 & $-1,00$ \\
$06 / 02$ & 1,00 & $-0,98$ & 0,98 & $-0,93$ & 0,93 & $-1,00$ \\
$13 / 03$ & 1,00 & $-0,93$ & 0,93 & $-1,03$ & \\
\hline
\end{tabular}

IE2=índice de estresse ao nitrogênio (GAMA et al., 2002); EA = eficiência agronômica do nitrogênio (CUI et al., 2009); IE1 = "Low N index" (FISCHER et al., 1983); IS=índice de sensibilidade ao estresse (FISCHER \& MAURER, 1978).

IE2 = stress index to nitrogen (GAMA et al., 2002); EA = agronomic efficiency of nitrogen (CUI et al., 2009); IE1 = "Low index N" (Fischer et al., 1983); IS = sensitivity to stress index (FISCHER \& MAURER, 1978).

Chantachume et al. ${ }^{13}$ descrevem, ainda, que valores deste índice maiores que as unidades indicam tolerância ao estresse, e menores, susceptibilidade. Araus et al. ${ }^{14}$ relatam a importância de técnicas e metodologias simples na avaliação a campo de genótipos e, Wu et al. ${ }^{2}$ sobre a correta mensuração da eficiência e a busca por metodologias mais práticas.

A herdabilidade da produtividade de grãos, considerando a média de todos os experimentos, foi de 54,4\% $\pm 6,1$ (Tabela 3 ) e, ao considerar os níveis de fornecimento de $\mathrm{N}$ em separado, os valores médios foram: 60,4\% $\pm 8,9$ (baixo $N$ ); $50,9 \% \pm 11,8$ (médio $N$ ) e $51,2 \% \pm 11,9$ (alto $N$ ). No entanto, o efeito do fornecimento de $\mathrm{N}$ foi mais visível nas datas de semeadura 11/12, 28/01 e 13/03 (avaliações no menor grupo de genótipos).

Presterl et al., ${ }^{15}$ na avaliação da produtividade de grãos em genótipos de milho, encontraram valores de herdabilidade variando de 0,36 a 0,94 (ambientes considerados de alto $N$ ) e de 0,41 a 0,88 (ambientes de baixo $\mathrm{N}$ ).

Emede e Alika ${ }^{16}$ observaram valores menores de herdabilidade em condições de baixo $\mathrm{N}$ em comparação as de alto $\mathrm{N}$, na maioria dos atributos avaliados e ainda, destacaram que a baixa variação genotípica está relacionada a baixa herdabilidade.
Para a seleção de genótipos eficientes, Bänzinger e Cooper ${ }^{17}$ e Weber et al. ${ }^{18}$ recomendam o uso de experimentos/condições em que os valores de herdabilidade dos atributos avaliados sejam altos.

A maioria dos coeficientes de correlação fenotípica foram significativos $(P<0,01 ; P<0,05)$, variando de 0,61 a 0,82, entre as produtividades de grãos obtidas nos diferentes níveis de fornecimento de $\mathrm{N}$ (Tabela 3). Exceções foram observadas entre a produtividade de grãos obtida nos níveis Baixo e Alto $N(r=0,39)$ e, Médio e Alto $N(r=0,51)$, quando os experimentos foram semeados em $11 / 12$. Os coeficientes de correlação genotípicos, quando no intervalo entre -1 a 1 , foram altos e positivos entre os valores obtidos nos níveis de $\mathrm{N}$ (Tabela 3).

Chen et al. $^{5}$ encontraram correlações da produtividade de grãos obtidas entre níveis de $\mathrm{N}$ em alguns experimentos e, em outros, não. Este fato, de acordo com os autores supracitados, pode estar relacionado a: experimentos com interação entre genótipo e ambiente (nitrogênio) não significativa; magnitude do estresse baixa e; redução de produtividade em torno de $10 \%$, do ambiente com alto suprimento de $\mathrm{N}$ para o de baixo. O primeiro fato relatado por Chen et al. ${ }^{4}$ foi observado nos dados obtidos em cada data de semeadura (dados não apresentados). 
Tabela 3. Herdabilidade $\left(\mathrm{h}^{2}\right)$ da produtividade de grãos e correlação entre os níveis de nitrogênio em avaliações com 12 genótipos de milho em quatro datas de semeadura $(11 / 12 ; 28 / 01 ; 06 / 02 ; 13 / 03)$ e 30 genótipos de milho em duas datas de semeadura (30/10; 06/03), safra 2012/13, Gurupi-TO, Brasil.

Table 3. Heritability $\left(h^{2}\right)$ of grain yield and correlation between nitrogen levels in evaluations with 12 corn genotypes in four sowing dates $(11 / 12 ; 28 / 01 ; 06 / 02 ; 13 / 03)$ and 30 genotypes corn in two sowing dates (30/10; 06/03), 2012/13 crop, Gurupi-TO, Brazil.

\begin{tabular}{|c|c|c|c|c|c|c|c|c|c|}
\hline \multirow{2}{*}{ Data de Semeadura } & \multicolumn{3}{|c|}{$h^{2}$} & \multirow{2}{*}{$\frac{\mathrm{BN} \text { vs. MN }}{\mathrm{rp}}$} & \multicolumn{2}{|r|}{ BN vs. AN } & \multicolumn{3}{|c|}{ MN vs. AN } \\
\hline & $\mathrm{BN}$ & $\mathrm{MN}$ & AN & & $\mathrm{rg}$ & $\mathrm{rp}$ & $\mathrm{rg}$ & $r p$ & $\mathrm{rg}$ \\
\hline $30 / 10$ & 69,24 & - & 67,06 & - & - & $0,66 * *$ & 0,98 & - & - \\
\hline 06/03 & - & - & 51,26 & - & - & $0,61^{* *}$ & 1,05 & - & - \\
\hline $11 / 12$ & 32,16 & 62,12 & 15,83 & $0,67^{*}$ & 1,23 & 0,39 & 2,46 & 0,51 & 2,50 \\
\hline 28/01 & 50,05 & 55,17 & 73,06 & $0,77^{* *}$ & 1,62 & $0,79 * *$ & 1,01 & $0,68^{*}$ & 1,31 \\
\hline 06/02 & 66,41 & 16,53 & 85,05 & $0,82 * *$ & 2,34 & $0,69 *$ & 0,96 & $0,67^{*}$ & 1,51 \\
\hline $13 / 03$ & 84,46 & 69,66 & 17,69 & $0,77^{* *}$ & 0,87 & $0,81^{* *}$ & 1,84 & $0,71^{* *}$ & 2,06 \\
\hline
\end{tabular}

BN-baixo N; MN-médio N; AN-alto N; rp-correlação fenotípica; rg-correlação genotípica. ** e * Significativo a p<0,01 e $p<0,05$, respectivamente, pelo teste $t$.

BN-low N; MN-medium N; AN-high N. rp-fenotypic correlation; rg- genotypic correlation. ${ }^{* *} \mathrm{e}^{*}$ Significant the $\mathrm{p}<0,01$ e $p<0,05$, respectively, by test $t$.

Combs \& Bernardo ${ }^{7}$ relatam que quando a correlação é negativa, desfavorável em algumas situações, a seleção para obter ganhos, num atributo, em dois ambientes deve ser evitada. Daros et al. ${ }^{20}$ relatam que quando a correlação (fenotípica/genotípica) é positiva os mesmos efeitos encontrados num atributo são observados no outro, ou ainda, quando se tem um atributo e dois ambientes. De acordo com Weber et al., ${ }^{18}$ a seleção indireta é possível quando existe alta correlação genética entre os ambientes e alta herdabilidade do atributo desejado num dos ambientes.

A seleção de genótipos superiores em condições de baixo fornecimento de $\mathrm{N}$ é uma alternativa que se relaciona ao contexto global pela busca de genótipos eficientes ${ }^{4,6}$ com melhor utilização do $\mathrm{N}^{21}$ com alta tolerância a estresses ${ }^{2}$.

\section{CONCLUSÃO}

Existe a possibilidade de usar ambientes com menor suprimento de $\mathrm{N}$ para a busca de genótipos eficientes para a produtividade de grãos. Por fim, o índice proposto por Fischer et al. (1983), "Low N index", é o mais adequado dentre os avaliados

\section{CONTRIBUIÇÃO DOS AUTORES}

1 - Planejamento, orientação, delineamento experimental e revisão final do trabalho/artigo

\section{Flávio Sérgio Afférri}

Joenes Mucci Peluzio 9

2 - Condução e avaliação do experimento, análise estatística e revisão final do trabalho/artigo

Edmar Vinícius de Carvalho

Michel Antônio Dotto

Eliane Aparecida Rotili

Lucas Alves de Faria

\section{REFERÊNCIAS}

1. SZÉLES, A. V.; MEGYES, A.; NAGY, J. Irrigation and nitrogen effects on the leaf chlorophyll content and grain yield of maize in different crop years. Agricultural Water Management, v.107, p.133144, 2012.

2. WU, Y.; LIU, W.; LI, X.; LI, M.; ZHANG, D.; HAO, Z.; WENG, J.; XU, Y.; BAI, L.; ZHANG, S.; XIE, C. LoWnitrogen stress tolerance and nitrogen agronomic efficiency among maize inbreds: comparison of multiple indices and evaluation of genetic variation. Euphytica, v.180, n.2, p.281-290, 2011.

3. MAKUMBI, D.; BETRÁN, J. F.; BÄZINGER, M.; RIBAUT, J. M. Combining ability, heterosis and genetic diversity in tropical maize (Zea mays L.) under stress and non-stress conditions. Euphytica, v.180, n.2, p.143-162, 2011.

4. CHEN, F.; FANG, Z.; GAO, Q.; YE, Y.; JIA, L.; YUAN, L.; MI, G.; ZHANG, F. Evaluation of the yield and nitrogen use efficiency of the dominant maize 
hybrids grown in north and northeast China. Science China Life Sciences, v.56, n.6, p.552-560, 2013.

5. ABE, A.; ADETIMIRIN, V. O.; MENKIR, A.; MOOSE, S. P.; OLANIYAN, A. B. Performance of tropical maize hybrids under conditions of low and optimum levels of nitrogen fertilizer application grain yield, biomass production and nitrogen accumulation. Maydica, v.58, n.2, p.141-150, 2013.

6. COMBS, E.; BERNARDO, R. Genomewide selection to introgress semidwarf maize germplasm into U.S. Corn Belt inbreds. Crop Sciences, v.53, n.4, p.1427-1436, 2013.

7. FANCELLI, A. L.; DOURADO-NETO, D. Produção de milho. 2.ed. Piracibaca-SP, Livroceres, 2004. 360p.

8. GAMA, E. E. G.; MARRIEL, I. E.; GUIMARÃES, P. E. O.; PARENTONI, S. N.; SANTOS, M. X.; PACHECO, C. A. P.; MEIRELES, W. F.; RIBEIRO, P. H. E.; OLIVEIRA, A. C. Combining ability for nitrogen use in a selected set of inbred lines from a tropical maize population. Revista Brasileira de Milho e Sorgo, v.1, n.3, p.68-77, 2002.

9. CUI, Z.; ZHANG, F.; MI, G.; CHEN, F.; LI, F.; CHEN, X.; LI, J.; SHI, L. Interaction between genotypic difference and nitrogen management strategy in determining nitrogen use efficiency of summer maize. Plant and Soil, v.317, n.1, p.267-276, 2009.

10. FISCHER, R. A.; MAURER, R. Drought resistance in spring wheat cultivars. I grain yield responses. Australian Journal of Agricultural Research, v.29, n.5, p.897-912. 1978.

11. FISCHER, K. S.; JOHNSON, E. C.; EDMEADS, G. O. Breeding and selection for drought in tropical maize. Mexico: CIMMYT, 1983.

12. CHANTACHUME, Y.; MANUPEERAPAN, T.; GRUDLOYMA, P.; TONGCHUAY, S.; NORADECHANON, S.; KONGTIAN, D.; LEON, C.; LOTRHOP, J. E. Selection for Low N Tolerance in the Thai Maize Breeding Program. México: CIMMYT, 1996.

13. ARAUS, J. L.; ELAZAB, A.; VERGARA, O.; SERRET, M. D.; ZAMAN-ALLAH, M.; CAIRNS, J. E. Advances in plant physiology and new technologies for maize and sorghum phenotyping under water stress. In: ABMS (Eds.). Congresso Nacional de Milho e Sorgo: Eficiência nas cadeias produtivas e o abastecimento global. Salvador, ABMS, 2014. p.29-36.

14. PRESTERL, T.; SEITZ, G.; LANDBECK, M.; THIEMT, E. M.; SCHMIDT, W.; GEIGER, H. H. Improving nitrogen-use efficiency in European maize: estimation of quantitative genetic parameters. Crop Sciences, v.43, n.4, p.1259-1265, 2003.

15. EMEDE, T. O.; ALIKA, J. E. Variation in agronomic characters among high and low nitrogen S2 maize (Zea mays $\mathrm{L}$ ) lines grown in high and low nitrogen environments. Maydica, v.57, n.2, p.139-146, 2012.

16. BÄNZINGER, M.; COOPER, M. Breeding for low input conditions and consequences for participatory plant breeding: examples from tropical maize and wheat. Euphytica, v.122, n.3, p.503-519, 2001.

17. WEBER, V. S.; MELCHINGER, A. E.; MAGOROKOSHO, C.; MAKUMBI D.; BÄZINGER M.; ATLIN, G. N. Efficiency of managed-stress screening of elite maize hybrids under drought and low nitrogen for yield under rainfed conditions in Southern Africa. Crop Sciences, v.52, n.3, p.1011-1020, 2012.

18. SEKER, H.; YAZICl, A.; UYSAL, P. Analysis of variability, heritability, and genetic advance in seed yield and related traits of orchardgrass (Dactylis glomerata L.) populations. Turkish Journal of Agriculture and Forestry, v.38, n.5, p.633-643, 2014.

19. DAROS, M.; AMARAL-JÚNIOR, A. T.; PEREIRA, M. G.; SANTOS, F. S.; SCAPIM, C. A.; FREITASJÚNIOR, S. P.; DAHER, R. F.; ÁVILA, M. R. Correlações entre caracteres agronômicos em dois ciclos de seleção recorrente em milhopipoca. Ciência Rural, v.34, n.5, p.1389-1394, 2004.

20. CIRILO, A. G.; DARDANELLI, J.; BALZARINI, M.; ANDRADE, F.H.; CANTARERO, M.; LUQUE, S.; PEDRO, H. M. Morpho-physiological traits associated with maize crop adaptations to environments differing nitrogen availability. Field Crop Research, v.113, n.2, p.116-124, 2009.

21. DoVALE, J. C.; FRITSCHE-NETO, R.; BERMUDEZ, F.; MIRANDA, G.V. Efeitos gênicos de caracteres associados à eficiência no uso do nitrogênio. Pesquisa Agropecuária Brasileira, v.47, n.3, p.385-392, 2012. 